\title{
Efficient Audio Noise Reduction System Using Butterworth Chebyshev and Elliptical filter
}

\author{
Aravind Kumar $\mathrm{M}^{*}$ and Manjunatha Chari $\mathrm{K}$ \\ Department of ECE, GITAM University, GITAM School of Technology \\ Hyderabad, Telangana, India \\ aravindkumarmtech@gmail.com
}

\begin{abstract}
Digital signal processing is widely used to manipulate, modify, enhance or filter signals such as speech, audio, image and telecommunication signal. Signals can be processed in the analog domain, but the digital domain offers high speed, better accuracy, greater flexibility increased storing capabilities and simple implementation. The audio signal noise reduction has become a fundamental area of research for many real world applications. There are two forms of filters one is fixed, and another one is tunable. Fixed filters measure those within which passband frequencies and stop band frequencies, square measure mounted whereas just in case of tunable filters, passband and stop band frequencies measured variable. These frequencies will be modified consistent with the need of the applications. Tunable digital filters are widely used in medical electronics, digital audio instrumentation, telecommunications and control systems. It is often the essential requirement for removal of noise from the audio signal.
\end{abstract}

Keywords: Fixed Filters, Tunable Filters, Audio Noise Reduction System

\section{Introduction}

It is vital for engineers of inserting frameworks, processors, and tools targeting video applications. The audio noise reduction system is the system that's accustomed take away the noise from the audio signal. Audio noise reduction systems are classified into two basic processes. Coming to the primary process is that the complementary sort that involves compressing the audio signal in a well-defined manner before it's recorded. The second method is that the single-ended or non-complementary sort that utilizes techniques to cut back the noise level already placed within the supply material-in essence a playback solely noise reduction system. This method is employed by the LM1894 integrated circuit, is designed for the reduction of hearable noise in nearly any audio source. Noise reduction is the method of removing noise in the signal. All analog or digital recording devices have traits that build them vulnerable to noise [15].

In device's mechanism or processing algorithms having noise. That will be white or random noise without coherence. There can be Active noise control (ANC), also called noise cancellation. Active noise reduction (ANR) may be a technique to reduce unwanted and unprocessed sound by the addition of a second sound specifically designed to cancel the first. The sound may be a pressure wave, or we may say the sound is that the analog signals that are processed consistent with their frequency, that is consisting of a rarefaction phase and compression phase. A noise-cancelling speaker is emitting a sound wave with an equivalent amplitude, however, with inverted phase (also called antiphase) to the original sound signal [9]. The waves are mixed to make the new wave, in an exceedingly process known as interference, and effectively cancel one another out an impression that is named phase cancellation.

Today's advanced active noise control is achieved usually through the employment of analog circuits or digital signal process. An associate adaptive algorithm, the square 
measure designed to investigate the waveform of the background noise, then supported the particular algorithmic rule generate a proof that may either phase shift or inverts the polarity of the original signal. This anti-phase is then amplified, and a transducer creates an acoustic wave directly proportional to the amplitude of the original waveform, making destructive interference [8] which can reduce a volume of perceivable noise effectively. The transducer emitting the noise cancellation signal could also be situated at the placement wherever sound attenuation is needed (e.g. The user's ear/any music/headphone sound) [7]. It requires a far lower power level for cancellation, however, is valid just for one user.

Types of Noises: Noises are classified based on the sources of noise or distortions and they include: i. An electronic noise was resembling thermal noise and shot noise, ii. Acoustic noise is emanating from moving, vibrating or colliding sources resembling revolving Machines, keyboard clicks, moving vehicles, rain and wind, iii. The electromagnetic noise that may interfere with the transmission and reception of image, voice, and knowledge over the spectrum, iv. With the presence of a voltage, Electrostatic noise generated, v. Fading and communicating distortion and vi. Lost data packets and Quantization noise due to network congestion. Signal distortion is that the term typically accustomed describes a systematic undesirable change in an exceedingly signal and refers to changes in an exceedingly signal from the non-ideal characteristics of the communicating, echo, signal fading reverberations, multipath reflections and missing samples [10]. Based on its frequency, time characteristics or spectrum, a noise method is more classified into many categories:

1. Band-limited white noise: Kind of like white noise, this is often a noise with a flat power spectrum and a limited bandwidth that sometimes covers the restricted spectrum of the device or the signal of interest. The autocorrelation of this noise has been looking like for-shaped.

2. White noise: strictly random noise has an associate impulse autocorrelation perform and a flat power spectrum. Noise contains all frequencies of equal power [2].

3. Colored noise: It's non-white noise or any broadband noise whose spectrum features a non-flat form. Examples for this are pink noise, brown noise, and autoregressive noise.

4. Narrowband noise: It's a noisy method with a narrow bandwidth resembling 50/60 $\mathrm{Hz}$ from the electricity supply.

5. Transient noise pulses: Encompass comparatively long length noise pulses are resembling burst noise, clicks, etc.

6. Impulsive noise: Consists of short-duration pulses of random amplitude, duration and time of prevalence.

The noise-related problem can be divided into non-additive noise and additive noise. The non-additive noise includes multiplier noise and convolution noise, which can be transformed into additive noise through homomorphism transform. The additive noise includes periodic noise, pulse noise and broadband noise related problems. There are many kinds of broadband noise, which may include heat noise, wind noise, quantization noise and all kinds of random noise such as white noise and pink noise. The statistical relationship between the noise and speech, i.e., uncorrelated or even independent noise and correlated noise (such as echo and reverberation). In accounts applications, noise from the surrounding environment severely reduces the quality of speech and audio signals. Therefore, basic linear filters are used to denoise the audio signals and enhance speech and audio signal quantity. As, we to increase the intelligibility of the overall speech perception quality such as Signal to Noise Ratio, Peak Signal to Noise Ratio, Mean Square Error and there to reduce the noise for noisy signals for removing noise

Active vs. Passive Noise Control

The topic Noise control is a full of passive or active suggests that by reducing sound 
emissions, typically for private comfort, environmental issues or legal compliance. Coming to active noise control; is sound reduction employing a power source. In the case of Passive noise control, the sound reduction can be taken place by noise-isolating materials resembling sound-absorbing tiles, insulation or a muffler instead of power source [1].

For low frequencies Active noise canceling is the best scheme. For higher frequencies, the spacing needs at no cost house, and zone of silence techniques become prohibitory. In acoustic cavity and duct based mostly systems, the many modes grow apace with increasing frequency, that quickly makes active noise control techniques unmanageable. Passive treatments become simpler at higher frequencies and infrequently give associate satisfactory resolution, while not the requirement for active control [3].

\section{Filters}

Filters measure the network that process signals in an exceedingly frequencydependent manner. The essential thought of a filter is explained by examining the frequency dependent nature of the impedance of capacitors and inductors. Filters have several practical applications. Let us discuss a simple, single-pole, low-pass filter (the Integrator) is usually accustomed stabilized amplifiers by rolling off the gain at higher frequencies wherever excessive phase shift could cause oscillations as shown in Figure 1. A simple, single-pole, high-pass filter is accustomed block DC offset in high gain amplifiers or single offer circuits [8]. Filters are accustomed separate signals, passing those of interest, and attenuating the unwanted frequencies. There square measure an oversized variety of texts dedicated to filter theory. An ideal filter has an associated amplitude response that's a unity (or at a fixed gain) for the frequencies of interest (called the pass band) and 0 all over (called the stop band). The Response at that frequency changes from pass band to stop band is observed because of the cutoff frequency.

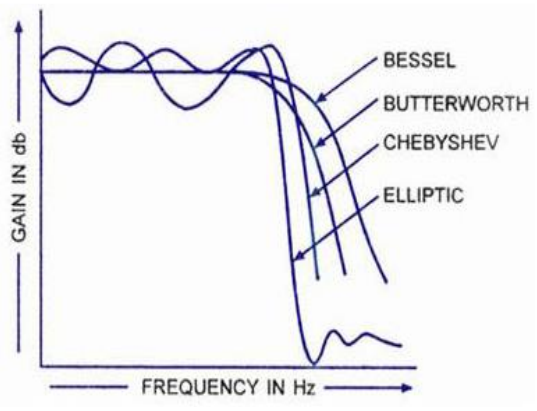

\section{Figure 1. Frequency Response Curve}

\section{Parametric methods:}

Parametric methods can yield higher resolutions than nonparametric methods in the case when the signal length is short. These methods use a different approach to spectral estimation, instead of trying to estimate the PSD directly from the data, they model the data as the output of a linear system driven by white noise and then attempt to estimate the parameters of that linear system.

The most commonly used linear systems are the all-pole model, a filter with all of its zeros at the origin in the z-plane. The output of the such a filter for white noise input is an auto regressive (AR) process. The noise-related problem can be divided into non-additive noise and additive noise. The non-additive noise includes multiplier noise and convolution noise, which can be transformed into additive noise through homomorphism transform. The additive noise includes periodic noise, pulse noise and broadband noise related problems.

Spectral analysis: 
The goal of spectral estimation is to describe the distribution (Over frequencies) of the power contained in a signal.Based on a finite set of data. Estimation of power spectra is useful in a variety of applications, including the detection of the signal buried in widebandwidth.

Welch's method:

An improved estimator of the PSD (Power Spectral Density) is governed proposed by Welch. The method consists of dividing the time series data into (possibly overlapping) segments, computing the modified periodogram of each segment and then averaging the PSD estimate. The result is Welch's PSD estimates the tool function Welch implements the Welch's method.

The averaging of modified periodograms tends to decrease the variable of the estimator relative to a single periodogram estimate of the entire data record. The combined use of short data records and nonrectangular windows results in reduced resolution of the estimator. There is a tradeoff between variable reduction and resolution. One can manipulate the parameters in Welch's method to obtain improved estimates relative to the periodogram especially when the SNR is low.

Problem Definition

The problem "Reduction of Audio Noise System Using Butterworth Chebyshev and Elliptic filter" is considered due to its present applications which include noise associated propagation problems in air handling systems in the industry, aircraft noise and tonal noise from electric power, and because of the isolation of vibration from noise is undesirable. The noises for the mentioned problem are additive and non-additive noise. The non-additive noise includes convolution noise and multiplicative noise, which might be reworked into additive noise using homomorphism transform. The additive noise includes pulse noise, periodical noise, and broadband noise related issues [12,6].

The noise generated by the engine is one reasonable periodic noise, whereas the one created from an explosion, bump, or discharge is pulse noise effect from the literature survey. Their area unit several forms of broadband noise, which can embrace heat, noise, quantization noise, wind noise and everyone forms of random noise akin to white and pink noise. The applied mathematical relationship between the noise and speech; i.e. uncorrelated or perhaps correlated noise (such as echo and reverberation) and independent noise. In acoustics applications, noise from the encompassing setting severely reduces the standard of speech and audio signals [13]. Therefore, totally different filters are a unit accustomed enhance speech and audio signal quality and denoised the audio signals. This paper titled as "Audio Noise reduction victimization Butterworth, Chebyshev \& Elliptical filters" aims for the subsequent objectives:

- The primary goal of a noise reduction system is profoundly passionate about the precise context and application. In some scenarios, maybe, we wish to extend the understandability or improve the sound perception quality [10].

- Study on the noise cancellation Techniques.

- According to a literature review Butter worth filter to Elliptical filter for Implementing the existing various techniques [5] [11] [8].

- Here Study and Analyze proposed results Being Obtained such as phase angle, frequency, time signal.

- Noise reduction technology was aimed at reducing unwanted surrounding noise and is implemented through different ways [4] [15].

\section{Objective of the Work}

- The objective of a noise reduction system is dependent upon the data length.

- Study of noise cancellation techniques

- To implement various types of filters Butterworth, Chebyshev, and Elliptic filters.

- Study and analyse the results such as signal spectrogram, Welch's power spectral density estimate, amplitude, and phase response, impulse response and step 
response.

- Calculating the SNR (Signal to Noise Ratio), PSNR (Peak Signal to Noise Ratio), MSE (Mean Square Error) and total time elapsed

- Noise reduction technology is aimed to reduce unwanted ambient noise.

\section{Methodology}

In this paper, we remove noise from the audio signal. It's based mostly upon GUI (graphical user interface) in MATLAB. It's a shot to any grasp the basics of MATLAB and validate it as a robust application tool. There are basically different files. Each of them consists of $\mathrm{m}$-file and Figure file. $\mathrm{m}$-file is the programmable file containing the data regarding the filter and Figure files is the way to analyse the given audio signal and enter the different types filters related data. During the present work, we'll first of all upload the input signal as a .wav format Waveform Audio File Format (WAVE, or all the more ordinarily known as WAV because of its filename extension), within the given window. In the GUI we will take the filter button and when click on the filter sound button, then a new window will open named filter sound. Then choose the desired filter for denoising and enter its various parameters, namely type, order, pass-band frequency, stop-band frequency, pass-band ripple and stop band ripple. Then click on ok than a new window filtered sound will open. This window shows filtered sound along with the graphs and various details about the filters like Transfer function, Step impulse and Frequency response.

\section{Algorithm for Filters:}

Step 1: Start the program.

Step 2: Load the wave signal that we want to denoised.

Step 3: Calculate the signal spectrogram and WELCH Power spectral density estimate.

Step 4: Apply filters.

Step 5: Choose different filter types.

Step 6: Butterworth filter, i.e Low pass filter, High Pass Filter, Bandpass filter, Band reject filter.

Step 7: Cheyvbyshev type-1 i.e Low pass filter, High Pass Filter, Bandpass filter, Band reject filter.

Step 8: Elliptic filter, i.e Low pass filter, High Pass Filter, Bandpass filter, Band reject filter.

Step 9: Choose the order of the filter and enter different types of frequency and press OK button.

Step 10: Analyse the filter response signal spectrogram and WELCH power spectral density estimate.

Step 11: Check the Amplitude response, Phase response, Impulse response, and Step response.

Step 12: Calculate the PSNR, SNR, MSE and the total time elapsed.

Step 13: Stop. 


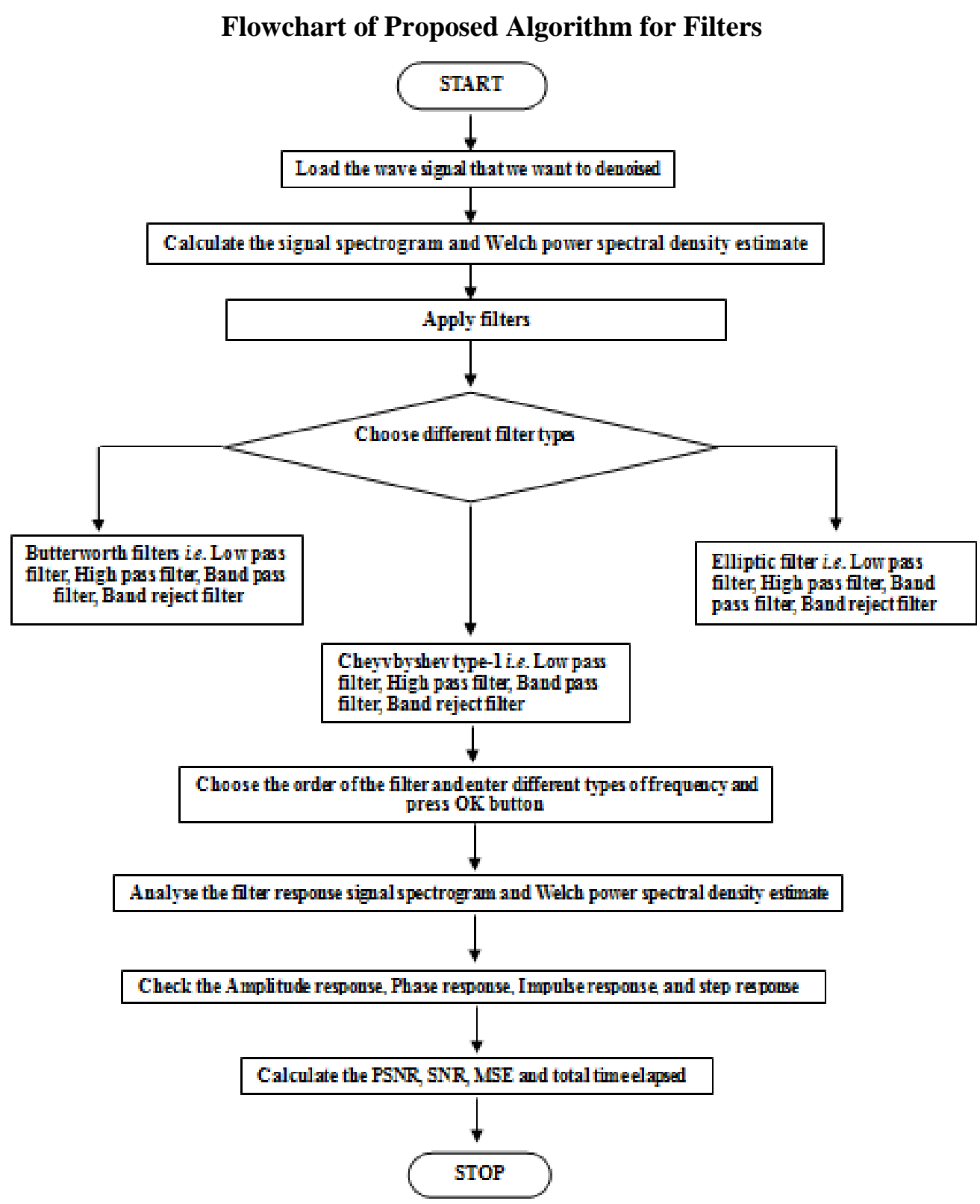

\section{Results and Discussions}

Figure 5.1 shows the initial window for uploading the input wave signal that we want to enhance. In this input, an audio signal is displayed Amplitude vs. Time and signal spectrogram is displayed Frequency vs. Time and Welch power spectral density estimate.

Figure 5.2 and Figure 5.3 shows the 8th order of Butterworth filter with low pass frequency, signal spectrogram and Welch power spectral density estimate. In this window, different values of frequency with a different order is used to enhance the signals.

Figure 5.4 shows the Amplitude and phase response. In Figure 5.5 shows the Impulse response. Figure 5.6 shows the step response of Butterworth filter.

Butterworth filter: 


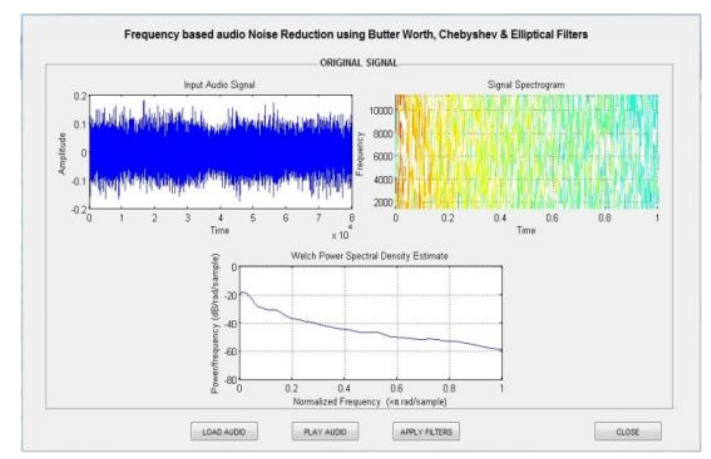

Figure 5.1. Initial Window for Uploading the Wave Signal

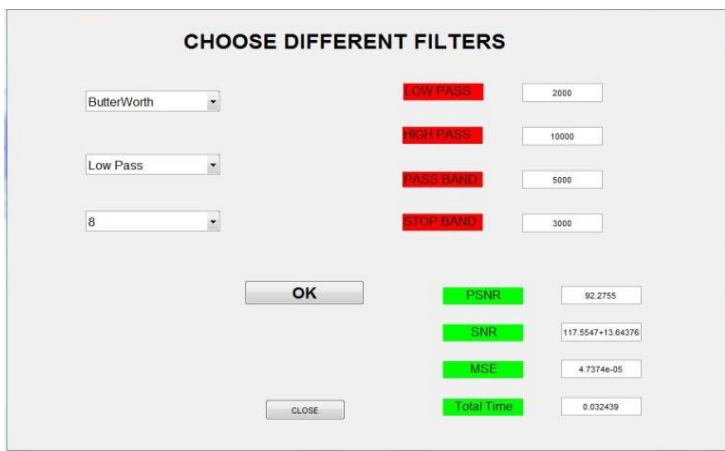

Figure 5.2. Butterworth Filter with Low Pass Frequency and 8th Orders

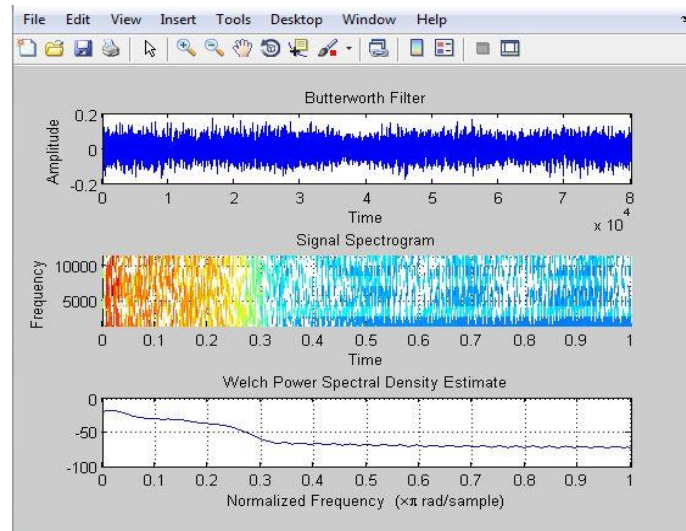

Figure 5.3. Spectrogram and Power Spectral Density after Applying a Butterworth Filter 


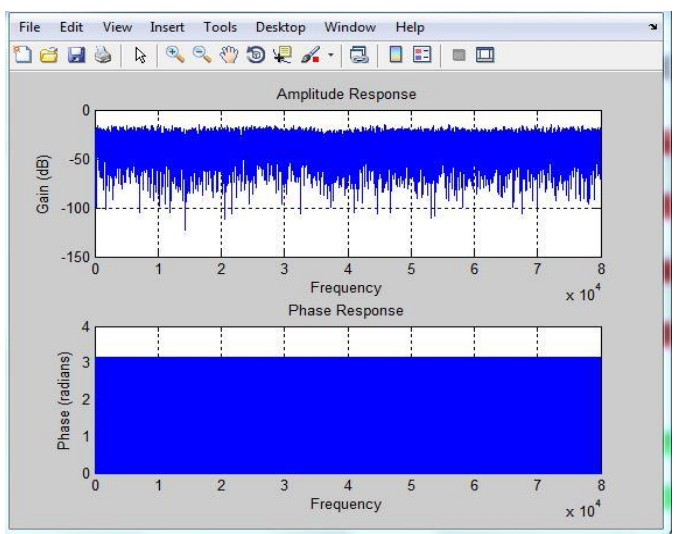

Figure 5.4. Amplitude and Phase Response

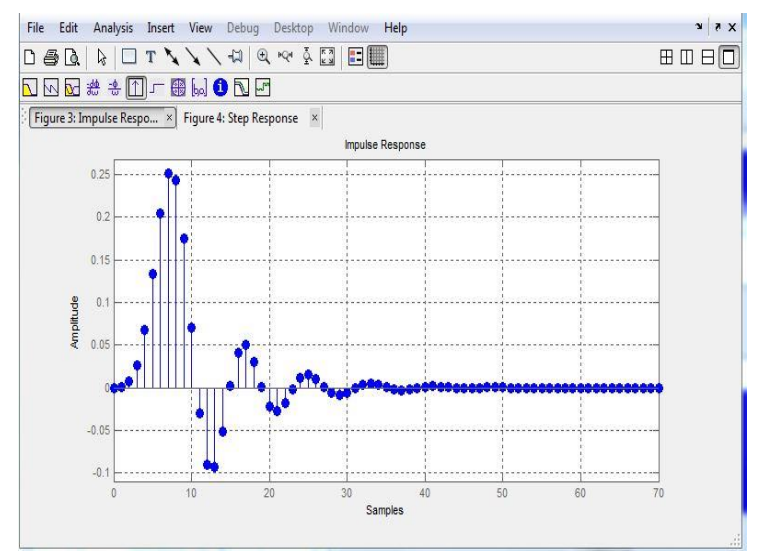

Figure 5.5. Impulse Response

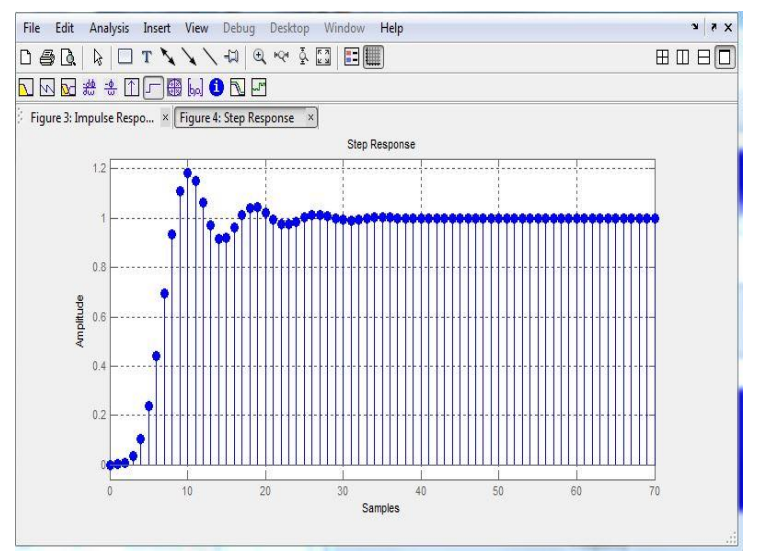

Figure 5.6. Step Response

Chebyshev Filter:

The Figure 5.7 and Figure 5.8 shows the initial window for uploading the input wave signal that we want to enhance. Selecting the order and the type of filter as Chebyshev filter.

The Figure 5.9 and shows the signal spectrogram and Welch power spectral density estimate. In this window, different values of frequency with the different order is used to enhance the signals. The Figure 5.10 shows the Amplitude and phase response.

The Figure 5.11 and Figure 5.12 shows the impulse response and step response of the filter. 


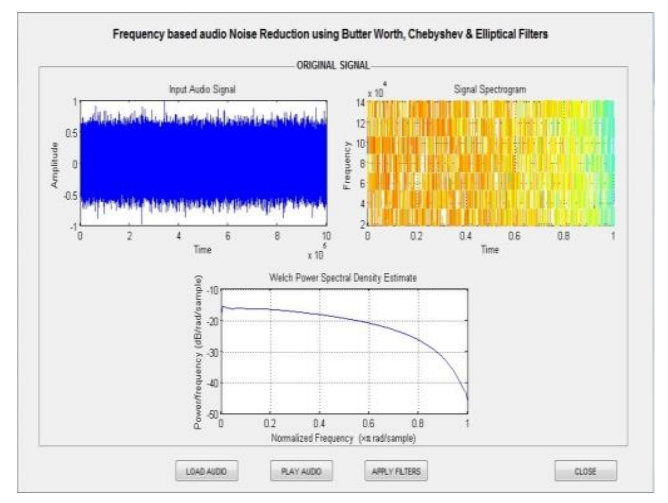

Figure 5.7. Initial Window for Uploading The Signal

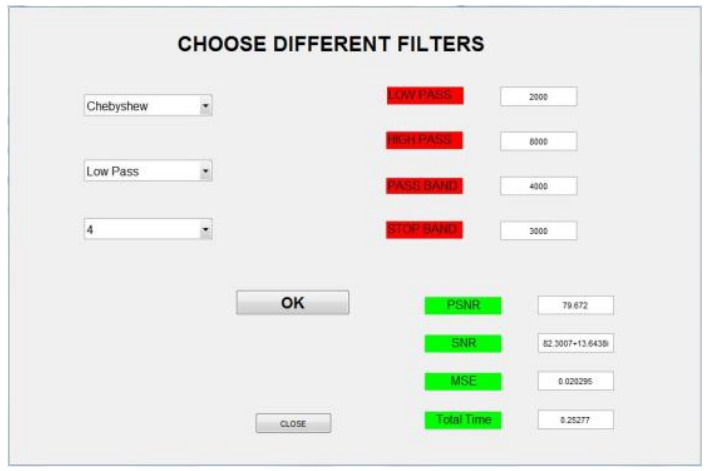

Figure 5.8. Selecting the Order and Type of the Filter

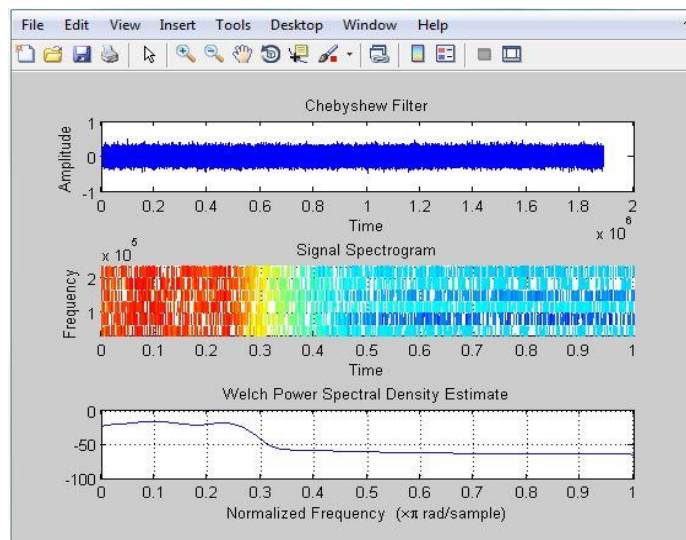

Figure 5.9. Spectrogram and Power Spectral Density after Applying to Filter 


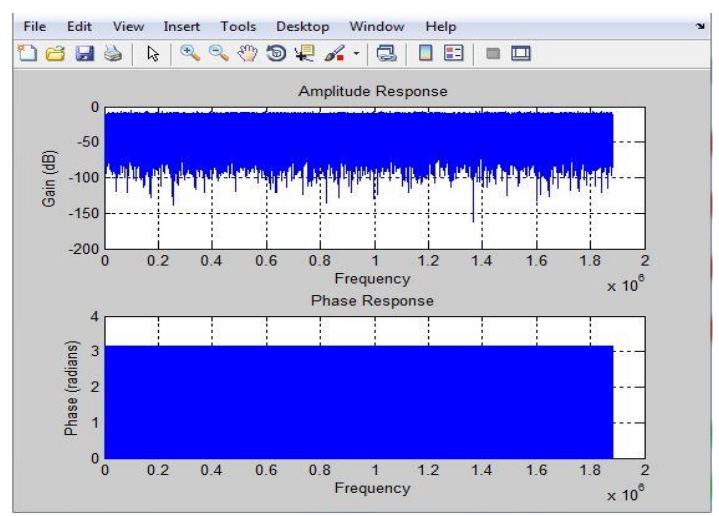

Figure 5.10. Amplitude and Phase Response

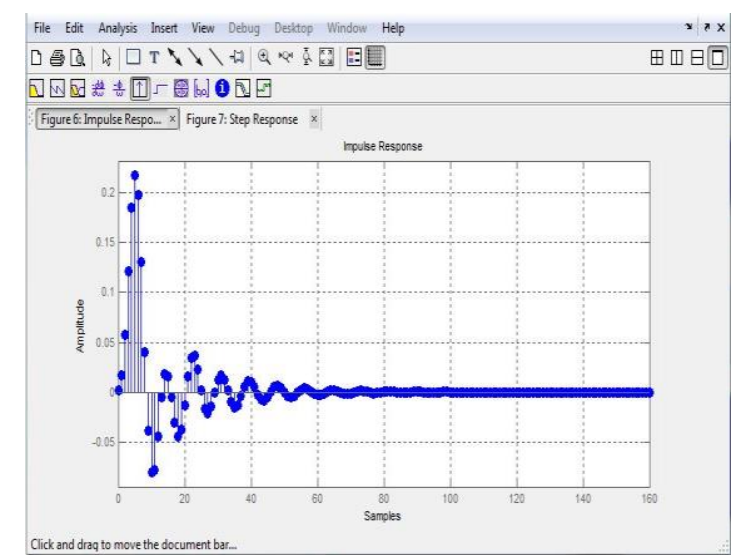

Figure 5.11. Impulse Response

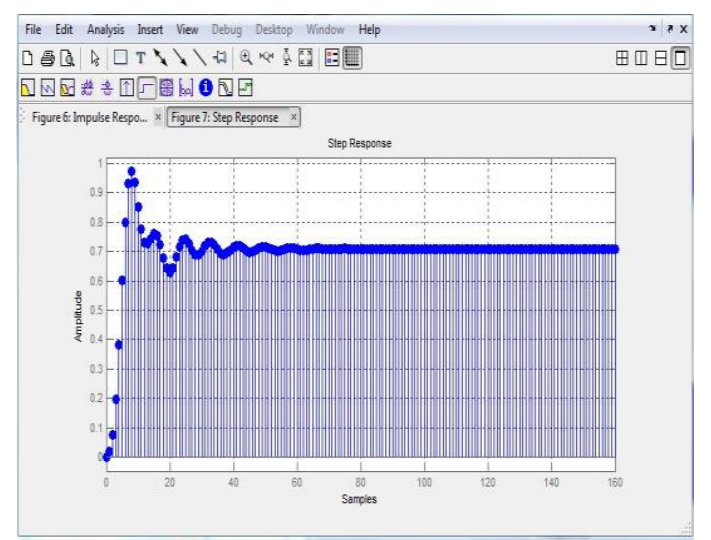

Figure 5.12. Step Response

Elliptic filter:

The Figure 5.13 and Figure 5.14 shows the initial window for uploading the input wave signal that we want to enhance. Selecting the order and the type of filter as Elliptical filter. The Figure 5.15 shows the Amplitude and phase response. The Figure 5.16 and Figure 5.17 shows the impulse response and step response of the filter. 


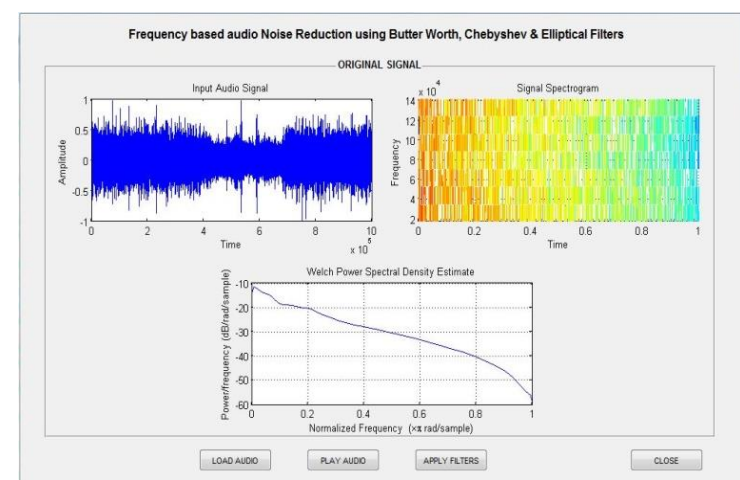

Figure 5.13. Initial Window for Uploading The Signal

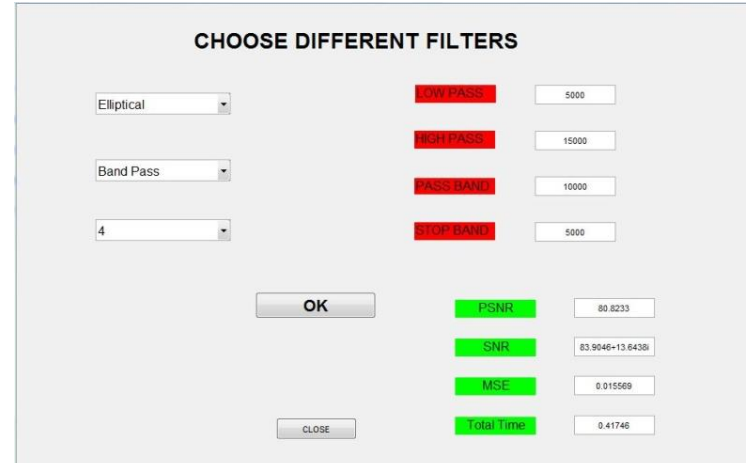

Figure 5.14. Selecting the Order and Type of the Signal

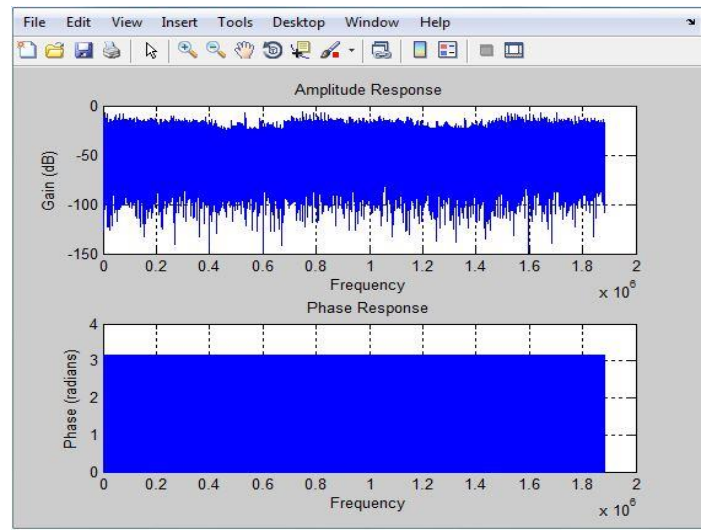

Figure 5.15. Amplitude and Phase Response 


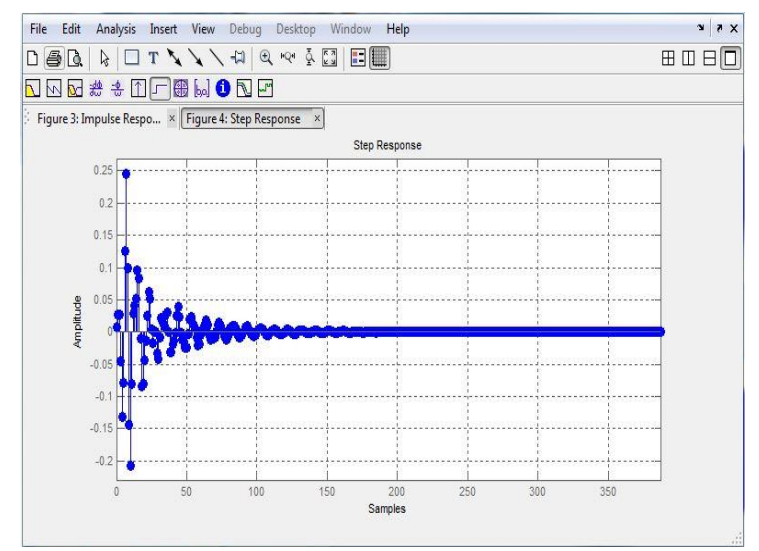

Figure 5.16. Impulse Response

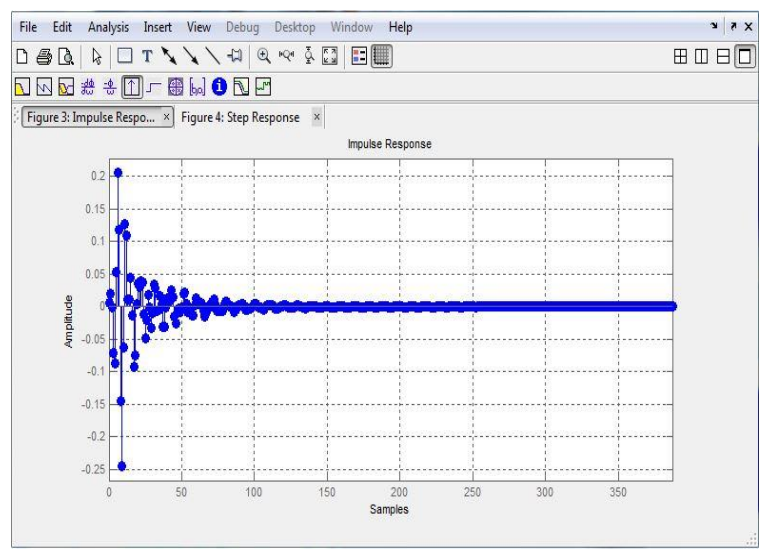

Figure 5.17. Step Response

Table 5.1. PSNR(Peak Signal to Noise Ratio) Value for different Filters

\begin{tabular}{|c|c|c|c|}
\hline $\begin{array}{c}\text { Name of } \\
\text { Signal }\end{array}$ & $\begin{array}{c}\text { Elliptical } \\
\text { Filter (12 }^{\text {th }} \\
\text { order) }\end{array}$ & $\begin{array}{c}\text { Butterworth } \\
{\text { Filter }\left(12^{\text {th }}\right.} \\
\text { order) }\end{array}$ & $\begin{array}{c}\text { Chebyshev } \\
\text { by Type-1 } \\
\text { Filter (12 } \\
\text { order) }\end{array}$ \\
\hline 1N.wav & 35.0010 & 40.5123 & 35.6001 \\
\hline 2N.wav & 35.0010 & 48.7001 & 35.6001 \\
\hline 3N.wav & 35.0010 & 35.521 & 35.6001 \\
\hline
\end{tabular}

Table 5.2. SNR(Signal to Noise Ratio) Value for different Filters

\begin{tabular}{|c|c|c|c|}
\hline $\begin{array}{c}\text { Name } \\
\text { of } \\
\text { Signal }\end{array}$ & $\begin{array}{c}\text { Elliptical } \\
\text { Filter }\left(12^{\text {th }}\right. \\
\text { order })\end{array}$ & $\begin{array}{c}\text { Butterworth } \\
\text { Filter }\left(12^{\text {th }}\right. \\
\text { order })\end{array}$ & $\begin{array}{c}\text { Chebyshev } \\
\text { by Type-1 } \\
\text { Filter }\left(12^{\text {th }}\right. \\
\text { order })\end{array}$ \\
\hline 1N.wav & 0 & -513.208 & 0 \\
\hline 2N.wav & 1.143 & -1417.75 & 5.1138 \\
\hline 3N.wav & 0 & -32.1753 & 0 \\
\hline
\end{tabular}




\section{Table 5.3. MSE(Mean Square Error) Value for Different Filters}

\begin{tabular}{|c|c|c|c|}
\hline $\begin{array}{c}\text { Name of } \\
\text { Signal }\end{array}$ & $\begin{array}{c}\text { Elliptical } \\
\text { Filter }\left(12^{\text {th }}\right. \\
\text { order })\end{array}$ & $\begin{array}{c}\text { Butterworth } \\
\text { Filter }\left(12^{\text {th }}\right. \\
\text { order })\end{array}$ & $\begin{array}{c}\text { Chebyshev } \\
\text { by Type-1 } \\
\text { Filter }\left(12^{\text {th }}\right. \\
\text { order })\end{array}$ \\
\hline 1N.wav & 68.4602 & 222.512 & 68.4402 \\
\hline 2N.wav & 68.4402 & 1425.33 & 68.4002 \\
\hline 3N.wav & 68.4002 & 73.588 & 68.4002 \\
\hline
\end{tabular}

\section{Table 5.4. Total Time Elapsed Value for Different Filters}

\begin{tabular}{|c|c|c|c|}
\hline $\begin{array}{c}\text { Name of } \\
\text { Signal }\end{array}$ & $\begin{array}{c}\text { Elliptical } \\
\text { Filter }\left(12^{\text {th }}\right. \\
\text { order })\end{array}$ & $\begin{array}{c}\text { Butterworth } \\
\text { Filter }\left(12^{\text {th }}\right. \\
\text { order })\end{array}$ & $\begin{array}{c}\text { Chebyshev } \\
\text { by Type-1 } \\
\text { Filter }\left(12^{\text {th }}\right. \\
\text { order })\end{array}$ \\
\hline 1N.wav & 0.0106016 & 0.0167216 & 0.0098894 \\
\hline 2N.wav & 0.036333 & 0.0340324 & 0.0094241 \\
\hline 3N.wav & 0.0082227 & 0.01508 & 0.0164941 \\
\hline
\end{tabular}

\section{Conclusion}

In this paper, the WELCH method is used to estimate the power spectral density. This method is used to find the seed of a signal by reducing the effect of noise. The quality of the estimated increase as the length $\mathrm{N}$ of the data increase which means the consistency. By using different types of filters, signal-to-noise ratio, Mean square error and Total Time Elapsed value are calculated.

\section{References}

[1] R. M. Ramli, A. O. A. Noor and S. A. Samad, "A Review of Adaptive Line Enhancers for Noise Cancellation", Australian Journal of Basic and Applied Sciences, ISSN 1991-8178, vol. 6, no. 6, (2012), pp. 337-352.

[2] E. Martin, "Audio denoising algorithm with block thresholding", Published in Image Processing On Line on YYYY \{MM \{DD.ISSN 2105-1232.

[3] B. JaiShankar1 and K. Duraiswamy "Audio denoising using wavelet transform", International Journal of Advances in Engineering \& Technology, ISSN: 2231-1963, (2012).

[4] C. M. Rao1 and B. S. Charles, "A Variation of LMS Algorithm for Noise Cancellation", International Journal of Advanced Research in Computer and Communication Engineering, ISSN (Print): 2319-5940, vol. 2, no. 7, (2013).

[5] B. J. Shankar and K. Duraiswamy, "Signal denoiser using wavelets and block matching process", Asian Journal of Computer Science and Information Technology, vol. 2, no. 1, (2012), pp. 1-3.

[6] J. Jebastine and B. SheelaRani, "Design and implementation of noise free Audio speech signal using fast block least Mean square algorithm", Signal \& Image Processing: An International Journal (SIPIJ), vol. 3, no. 3, (2012).

[7] G. Yu and S. Mallat, "Audio Denoising by Time- Frequency Block Thresholding”, IEEE Transactions on Signal Processing, vol. 56, no. 5, (2008).

[8] R. Sharma and V. P. Pyara, "A Robust Denoising Algorithm for Sounds of Musical Instruments Using Wavelet Packet Transform”, Circuits and Systems, Published Online November 2013, vol. 4, (2013), pp. 459-465.

[9] M. E. Abdulmunim and R. F. Abass, "Novel Video Denoising Using 3-D Transformation Techniques", International Journal of Engineering and Advanced Technology (IJEAT) ISSN: 2249 - 8958, vol. 2, no. $5,(2013)$.

[10] K. P. Obulesu and P. U. Umar, "Implementation of time, frequency block thresholding algorithm in audio noise reduction", ISSN: 2278 - 7798 International Journal of Science, Engineering and Technology Research (IJSETR), vol. 2, no. 7, (2013). 
[11] S. N. Sampat and C. H. Vithalani, "Customized Neighborhood Threshold Speech Denoising Using Wavelet Transform Based on Filter Bank Method", International journal of darshan institute on engineering research \& emerging technologies, vol. 2, no. 1, (2013).

[12] R. Aggarwal, "Noise Reduction of Speech Signal using Wavelet Transform with Modified Universal Threshold", International Journal of Computer Applications (0975 - 8887), vol. 20, no. 5, (2011).

[13] K. Siedenburg and M. D"orfler, "Audio Denoising by Generalized Time-Frequency Thresholding", aes 45th international conference, Helsinki, Finland, (2012).

[14] B. Anitha, S. Bachu and C. Sailaja , "System Identification and Echo Canceller with Adaptive Filtering Algorithms", i-manager's Journal on Digital Signal Processing (JDP), ISSN (O):2322-0368, vol. 3, no. 3, (2015), pp. 13-17. 